\title{
Decreasing malaria transmission in Mojo Health Center, Ethiopia: Shifting burden of the disease and its implication for malaria elimination
}

Andargie Abate ( $\sim$ andargie_abate@yahoo.com )

Bahir Dar University College of Medical and Health Sciences https://orcid.org/0000-0003-3987-3119

Lemu Golassa

Addis Ababa University Aklilu Lemma Institute of Pathobiology

\section{Research}

Keywords: Ethiopia, Mojo, Malaria, Prevalence, Retrospective

Posted Date: May 11th, 2021

DOl: https://doi.org/10.21203/rs.3.rs-493774/v1

License: (c) (1) This work is licensed under a Creative Commons Attribution 4.0 International License.

Read Full License 


\section{Abstract}

Introduction

Malaria continues to strike hardest against the public health and economic development in Ethiopia. Its transmission tends to be highly heterogeneous within or between years, and from area to area. Thus, this study was aimed to determine the trend prevalence of malaria in Mojo health center, East Shoa Zone, Ethiopia.

Methods

A retrospective study was conducted in Mojo health center, East Shoa zone, Ethiopia from February to March, 2021. Malaria cases and related data reported between 2016 and2020 were carefully reviewed from laboratory registration logbooks. The collected data was analyzed using SPSS 25 software.

Results

A total of 19, 106 blood films were examined from malaria suspected patients. The overall microscopically confirmed prevalence of malaria was $4.2 \%$. P. vivax was the predominant species accounting $76.2 \%$ of positive samples. Malaria cases declined from 259 in 2016 to 77 in 2020 . The proportion of malaria was higher among males $(64.8 \%)$ than females $(35.2 \%)$. Higher malaria cases were observed from the age group 15-24 years followed by the age group of 25-34. Malaria cases were at a peak level from September-November and lowest from December-February.

Conclusion

Despite declining trend of malaria prevalence was observed, malaria still remains a public health burden in the area. The high burden of malaria among reproductive age group, males, and during cultivation season reflects its impact on health and economic development. Shifting of $P$. falciparum to P.vivax related malaria should get an attention during prevention and control strategies for the successful progress of malaria elimination programme.

\section{Introduction}

Despite being preventable and treatable, malaria continues to be one of the global deadly diseases contributing a significant impact on public health and economic development. An estimated 229 million cases and 409000 deaths were reported in 2019. The disease remains as a substantial health burden particularly in African countries composing $94 \%$ of the worldwide malaria cases and deaths [1].

In over a decade, remarkable achievements in malaria control were made. The global malaria case incidence (cases per 1000 population at risk) reduced by less than 2 , and the mortality rate (deaths per 100000 population at risk) from 12 in 2015 and 10 in 2019 indicating a slowing of decline on the rate of cases and deaths since 2015 [1]. Nevertheless, malaria control programmes in the past have prioritized to 
focus on $P$. falciparum malaria due to its consideration as more pathogenicity. This priority dangerously minimized the clinical and public health importance of P.vivax. As many countries progress towards the elimination of the disease, it is imperative to give a focus on $P$. vivax since $P$. vivax imposes a challenge that may hinder the achievement of the global malaria elimination program by $2030[2,3]$.

There is an increasing report that P.vivax can be associated with severe disease and carries a huge health, and socio-economic burden. P.vivax has evolved an ability to sustain transmission due to its capable of relapse, resurgence due to lack of radical cure, transmission to a larger range of vectors at wider range of temperature, and its genetic diversity. Currently reported non-Duffy antigen invasion pathways and emergence of resistant to the first line drug called chloroquine present huge challenges to the ambitious goal of malaria elimination $[2,4,5]$.

Ethiopia is also shared the globally estimated $82 \%$ ( 11.7 million cases) of P.vivax burden comes from four high-burden countries[6]. Thus, malaria continues to strike hardest against the health of population in Ethiopia. About $60 \%$ of the population lives in malarious areas, and $68 \%$ of the country's landmass is favorable for malaria transmission associated with altitude and rainfall. The peak of malaria incidence follows the main rainfall season (July to September) each year. However, many areas in the south and west of the country have a rainfall season starting earlier in April and May or have no clearly defined rainfall season. As a result, malaria transmission tends to be highly heterogeneous within or between years, and from area to area [7-10].

There was a fluctuating trend of malaria within the last decade showing a significant reduction of malaria in 2014/15 compared to the previous 2013/2014 [11]. This declining report has been supported by the national malaria indicator survey 2015 showing the reduction of the prevalence from $1.3 \%$ in 2011 to $0.5 \%$ in 2015 [7]. Furthermore, Deribew and his colleagues have also documented the decrement of malaria new cases and deaths from 2.8 million to 621,345 cases, and 30,323.9 to 1561.7 deaths in 1990 and 2015, respectively [12]. A previous retrospective studies have also reported malaria reduction [1315].

However, the report on decline of malaria is not conclusive that public health emergency management (PHEM) has shown an increased malaria transmission[11], and have no clearly defined situation of malaria [10]. It might be due to several factors including the resistance of the parasite to antimalarials and vectors to insecticides, and complexity of control process. In addition, the country has an interaction of variable winds, seasonal rains and ambient temperatures causing diverse micro-climates with low population immunity accelerating focal malaria transmission [11].

The fact that the prevalence of malaria is expected to shift over the years informing an understanding of the contextual diversity of malaria prevalence within each site to identify the optimal interventions, and to deliver according to the site specific situation of the disease. Therefore, determining epidemiological trends of malaria cases from specific health facility is valuable to measure progress towards malaria elimination. Thus, this study was aimed to determine the trend prevalence of malaria in Mojo health center, East Shoa Zone, Oromia region Ethiopia. As a result, results from this study will be useful in 
understanding and improving the interventions and in providing the information for development of new strategies for burden reduction and elimination of malaria in the country.

\section{Methods And Materials}

\section{Study setting, and design}

An institution based retrospective cross-sectional study was conducted in Mojo health center located in Mojo town from February to March, 2021. The Town is located in East Shoa zone, Oromia regional state at a distance of $70 \mathrm{~km}$ from Addis Ababa (capital city of Ethiopia), and $25 \mathrm{~km}$ from Adama town (Fig. 1). The town has an altitude range of $1730-1890 \mathrm{~m}$ above sea level and characterized by its mean annual temperature of $16-17^{\circ} \mathrm{C}$, and mean annual rainfall of $896 \mathrm{~mm}$. It has a latitude and longitude of $08^{\circ} 37^{\prime}$ North and $39^{\circ} 07^{\prime}$ East, respectively. According to the Central statistical agency of Ethiopia' projection carried out in 2015 , the population of the town was 43,500 . It has two health centers and one hospital. Among these, Mojo health center was chosen for this study since most of the town's population need to visit this health center due to its long duration of service provision than the two health institutions (Key informants). The town is malarious. Malaria is the most prevalent and seasonal disease in the area where both P.vivax and $P$. falciparum exist.

\section{Study population}

The study populations were all malaria suspected individuals who had visited the health center and blood film tested from January 2016 to December 2020.

\section{Eligibility criteria}

Data such as malaria cases diagnosed in months and years, types of Plasmodium species identified, and socio-demographic data (age and sex) were included in the analysis regardless of age, pregnancy and other infection status. Any missing data in the inclusion criteria were excluded.

\section{Data collection and quality control}

Five years malaria cases and related data were collected from Mojo health center laboratory logbooks using well developed check list. The check list consisted of year and month of visit, sex, age, blood film status (+/-), and detected Plasmodium species type. Data was collected by experienced medical laboratory technicians. In the health center, microscopic blood film examination was used as a golden standard to confirm the presence of Plasmodium parasite and identification of the species as recommended by WHO's protocol. The blood film examination for malaria parasite detection was performed according to standard operating procedure (SOP). The laboratory technologists or technicians who performed the microscopic examination were well trained in malaria microscopy. Microscopy examination was used exclusively for Plasmodium species detection throughout the study period (20162020). The data extraction process was followed up, and data were checked for completeness before analysis. Incompletely registered data were excluded from analysis. 


\section{Data processing and analysis}

The collected data was coded, cleaned and entered to Epi-data and exported to SPSS 25 software for analysis. The findings were presented using frequency, tables and figures for relevant data. Descriptive statistics were performed to determine the frequencies and percentages of overall malaria prevalence, and trends of malaria transmission in terms of years, seasons, plasmodium species, sex and age. Chisquare test was employed to determine the association of malaria burden by sex and age groups. Pvalue $<0.05$ was considered statistically significant in this study.

\section{Results}

\section{Characteristics of the study population}

During the study (January 2016 to December 2020), a total of 19, 106 blood films from malaria suspected patients were examined to detect the presence of Plasmodium parasite. Lower (7.7\%) number of blood films were examined in 2020. Majority of the study participants were females (54.5\%). Near to thirty percent of the participants were in the age group of 15-24 years (Table 1).

Table 1

Total number of examined blood films distribution with regard to age, sex and year in Mojo health center from 2016 to 2020

\begin{tabular}{|lll|}
\hline Variables & Category & Frequency (\%) \\
\hline Sex & Male & $8699(45.5)$ \\
\cline { 2 - 3 } Age & Female & $10407(54.5)$ \\
& $<5$ & $3208(16.8)$ \\
& $5-14$ & $2302(12.0)$ \\
& $15-24$ & $5747(30.1)$ \\
\hline & $25-34$ & $4237(22.2)$ \\
& $>=35$ & $3612(18.9)$ \\
Year & 2016 & $3965(20.8)$ \\
& 2017 & $4443(23.3)$ \\
& 2018 & $4671(24.4)$ \\
\hline 2019 & $4550(23.8)$ \\
\hline 2020 & $1477(7.7)$ \\
\hline
\end{tabular}




\section{Overall prevalence and annual trend of malaria}

The overall microscopically confirmed prevalence of malaria was $4.2 \%(793 / 19106)$. P. vivax was the predominant species in the study area in each year accounting $76.2 \%$ followed by $P$. falciparum (22.8\%) and mixed infections (1.0\%). Malaria cases had shown decrement from 259 in 2016 to 77 in 2020 (Fig. 2). When the proportion was considered, the prevalence was declined from 6.5\% (259/3965) in 2016 to $2.3 \%(104 / 4550)$ in 2019 , however it was increased to $5.2 \%(77 / 1477)$ in 2020 , and the variation was statistically significant $(P<0.0001)$ (Fig. 3).

\section{Malaria Distribution in relation to Age and Sex}

Out of 793 malaria cases in 5 years, 514 (64.8\%) and 279 (35.2\%) were detected from males and females, respectively (Table 2 ). According to five years record of malaria cases, proportion of malaria was higher among males than females in all five years (Fig. 4). Regarding morbidity of malaria by age group, malaria was reported in all age groups and ranged from 7.4-37.3\% out of recorded malaria cases within the five-year record in the study area. Relatively higher malaria cases (296 out of the total 793 malaria cases) was recorded from age group 15-24 years old followed by age group of 25-34 (211 out of 793 total malaria cases) (Table $2 \&$ Fig. 5). The difference in the prevalence of malaria among sex and age difference was statistically significant $(P<0.0001)$.

Table 2

Recorded Malaria Distribution in relation to Age and Sex in Mojo health center from 2016 to 2020

\begin{tabular}{|lll|}
\hline Variables & Category & Frequency (\%) \\
\hline Sex & Male & $514(64.8)$ \\
\cline { 2 - 3 } Age & Female & $279(35.2)$ \\
& $<5$ & $59(7.4)$ \\
\hline $5-14$ & $107(13.5)$ \\
& $15-24$ & $296(37.3)$ \\
& $25-34$ & $211(26.6)$ \\
& $>=35$ & $120(15.1)$ \\
\hline
\end{tabular}

\section{Seasonal Distribution of Malaria}

The seasonal distribution of malaria cases for the four seasons was analyzed. Malaria cases were reported in all months and seasons with some fluctuating trend. The peak in malaria cases occurred in July followed by November which is harvesting season during and after the main rainfall (Fig. 6). The highest cases of malaria (266 out of the total 793 cases) were observed during autumn (September- 
November), while the lowest cases (134 out of the total 793) were during winter (December-February). P.vivax infection was high in the summer and low in winter, while $P$. falciparum infection was high in the autumn and low in spring (Fig. 7).

\section{Discussion}

Malaria affects the lives of almost all people living in the area of Sub Saharan African countries where people at risk of the disease live in areas of relatively stable and seasonal malaria transmission[1]. In Ethiopia, malaria continuous as major public health problem despite relatively low prevalence compared to some African countries where malaria is endemic. Unstable malaria transmission patterns make the country prone to epidemics $[7,16]$. However, the country has planned to eliminate malaria from the whole country which needs sub-national elimination before nationwide elimination can be achieved. This is because the decrease in malaria burden is not uniform in the country [17] which is imperative to understand the contextual diversity of malaria prevalence within each site. Thus, this study determined epidemiological trends of malaria cases in specific health facility.

The current study found that the total number of microscopically confirmed malaria cases were 793 with a proportion rate of $4.2 \%$. It was markedly lower than previous studies conducted out of Ethiopia elsewhere (25.9\%-30.3\%) [18, 19], and in several parts of the country such as in Dembia $(21.8 \%)$ [10], Dembecha (16.34\%) [20], Guba (51.04\%) [15], Sibu Sire (20.07\%) [21], Arjo-Didessa sugar development site (33.4\%) [22], Harari (46.9\%) [23], Chalia district (7.55\%) [24], Abeshge (33.8\%) [25], and Bale Zone $(66.7 \%)$ [26]. The overall prevalence of the current study was also relatively lower than the findings from other parts of Ethiopia like Northwest Tigray (6.96\%) [8], Wereta (5.4\%) [27], Kombolcha (7.52\%) [13], Ataye (8.4\%) [28], Halaba (9.5\%) [9], Wolkite (8.56\%) [29] and Arsi Negelle (11.45\%) [30]. However, this study found higher prevalence of malaria relative to studies carried out abroad such as Panama (1.22\%) [31] and India (1.3-3.07\%) [32, 33].

These differences might be due to the fact that the quality of microscopic diagnosis is highly influenced by slide preparation and staining methodology, microscopist skill and quality of microscope [34]. The difference in time variations of the studies might also brought the difference on the overall prevalence of malaria since malaria is being declined from time to time as supported by the global[1], and national report of decreasing malaria prevalence since 2000 [17]. Moreover, the variations in malaria prevalence might also be due to geographical locations because of the fact that population awareness about malaria and application of intervention activities vary from area to area. Variation in sociodemographic status and utilization of intervention in the same locality also influence the prevalence as documented by previous studies conducted elsewhere [35-39].

In addition, the low prevalence of malaria in the current study could be due to the variation in species showing that $P$. vivax was the more predominant species in this study than $P$. falciparum in another studies listed above. This is due to the fact that the parasite density in $P$. vivax is typically lower than $P$. falciparum increasing the risk of false negative microscopy diagnosis with acute P.vivax malaria [34, 40, 
41]. The thought was also supported by the review stated that microscopists correctly identified only $63 \%-86 \%$ of confirmed P.vivax positive blood films [40]. Another review also summarized that an average of $67 \%$ of $P$. vivax infections were reported as not detected by microscopic examination [42] masking the actual burden of P.vivax related malaria. This might be approved by the reporting of lower prevalence of P.vivax related malaria from studies done in Panama (1.22\%) [31], and India (3.07\%) [33] where P. vivax was the predominant species.

The results of the present study revealed that a sharply declined fashion of malaria cases was observed during the last five years. This finding was consistent with the worldwide decreasing malaria burden in which 1.5 billion malaria cases have been averted from 2000 to 2019 where most of the averted cases $(82 \%)$ were in the WHO African regions including Ethiopia [1].A significant decline of malaria has been also reported in Ethiopia since 2000 [17]. The consistent reduction of malaria case in the current study was also concurred with the result of previous studies conducted in Vietnam [43], and other parts of the country such as Wolkite [29] and Jimma [44].

This might be due to the increased scaled-up of malaria control interventions by national malaria control programme of Ethiopia such as prompt diagnosis, community awareness creation, increased accessibility of long-lasting insecticide-treated nets (LLITNs) and high coverage of indoor residual spraying (IRS) [16]. This conclusion is further supported by the study indicating the reduction of malaria cases and deaths after implementing intervention [45]. This is also confirmed by the national malaria elimination roadmap proving that sustained high coverage of such interventions had reduced malaria morbidity by more than $50 \%$ between 2001 and 2011 [17]. The current progressive decline in annual malaria cases is suggestive of good national progress towards achieving of 2030 goals to reduce malaria incidence and mortality by at least $90 \%$ [17].

In contrary to the national profile of Plasmodium species in which $P$. falciparum is a dominant species $[10,17,23,26,46]$, $P$. vivax accounted for $76.2 \%$ and was predominately reported in the current study. However, in line with the current study, P.vivax was reported as predominant species in studies done in Arsi Negelle [30] and Wolkite [29]. It could be due to the fact that malaria control programmes in the past had prioritized to focus on $P$. falciparum malaria because of its consideration as more pathogenicity, and easier to detect and treat $[3,6]$. In addition, it could be due to the emergence of drug resistant [40], the parasite's ability to transmit early in the course of the disease [47], production of gametocytes transmitted more efficiently $[48,49]$, and a relapse from dormant liver stages $[6,50,51]$. This increment of the prevalence of P.vivax causing malaria with the decline of $P$. falciparum related malaria might also be supported by the fact of reporting Duffy independent invasion and genetic diversity of P.vivax leading to generation of new parasite strains that can translate into parasite's greater adaptability to new challenges for treatments and control measures [47]. Previous meta-analysis found high risk of P.vivax malaria after treatment of P.falciparum malaria [52].

In the present study, distribution of malaria cases was observed in both gender and all age groups of the population throughout the study period. Nevertheless, males were more affected than females which is 
consistent with studies conducted in other parts of Ethiopia[8-10, 15, 20,22, 26] and out of Ethiopia in France [53] and Kuwait [19]. This gender difference in malaria prevalence could be due to the fact that males frequently bitten by mosquitoes due to the behavioral differences of males' working outdoors, little chance of sleeping under nets, travel to endemic sites for work, and dressing norms are more likely than females to expose their bodies increasing their chance of mosquitoes' bite [54]. Moreover, hormonal differences might play the role in such a way that testosterone was associated with decreased adaptive immunity against malaria [55]. Briggs and the colleagues have also thought that females cleared their infections at a faster rate than males [56] which might support the lower prevalence of malaria among females in the current study.

The present study had observed higher prevalence of malaria among age group of 15-24 years old than younger children and older adults which is in line with studies done in other parts of Ethiopia[10, 15, 28]. This might be due to the fact that productive age groups frequently engaged in outdoor activities. Surprisingly, lower prevalence of malaria was observed in children under 5 years of age which might be because of their less likely exposure to mosquito bite, and sleeping under mosquito nets.

The current study observed malaria cases in all months and seasons throughout the study period. It might indicate that malaria transmission is determined by socio-economic and biological factors such as mosquito control measures, population immunity, governmental policy and drug resistance have also contribution on malaria transmission and prevalence. In spite of that, the peak in malaria cases occurred in July followed by November which is harvesting season during and after the main rainfall. The highest cases of malaria were detected during autumn (September-November) which was in consistent with the findings of the studies carried out in Dembia [10], Dembecha [20], Northwest Tigray [8], Ataye [28], Guba [15], Jimma [44], and Harari [23]. It might be due to the heavy rains creating suitable environment for the breading of Anopheles mosquitoes. In addition, it could be related to the formation of stagnant water after the heavy rain season, favorable temperature, and high vegetation density for mosquito breeding. The coinciding high prevalence of malaria with these cultivation months has a deleterious socioeconomic effect on productivity and development in the country.

\section{Conclusion}

The declining trend of the overall malaria prevalence was observed in the study area which is suggestive of good progress towards achieving of 2030 goals of malaria elimination. However malaria still remains a public health burden in the area affecting $4.2 \%$ of the population. P. vivax is the dominant species in the study area reflecting the shift of $P$. falciparum to P.vivax related malaria burden challenging the success of ongoing malaria elimination programme. The reproductive age group and males were more affected by the burden of malaria, and this was more common during cultivation season impacting on health and economic development. Therefore, malaria control and elimination programmes should be strengthened for further reduction of the burden of malaria particularly among highly affected groups. There is also a need for intensifying the prevention and control strategies on $P$. vivax. 


\section{Abbreviations}

CDC: Center for Diseases Control and Prevention; EPHI: Ethiopian Public Health Institute; FMOH: Federal Ministry of Health; IRS: Indoor Residual Spraying; LLITNs: Long-Lasting Insecticide-Treated Nets; SPSS: Statistical Package for the Social Science; WHO: World Health Organization; UNDP: United Nations Development Programme; USAID: United States of America Department of State; WWARN: Worldwide Antimalarial Resistance Network

\section{Declarations}

\section{Authors' contributions}

AA and LG conceived the study. AA collected and analyzed the data, and wrote the first draft of the manuscript under supervision of LG. AA generated a map. AA and LG critically reviewed the manuscript. Both authors read and approved the final manuscript.

\section{Acknowledgements}

The authors are grateful to Aklilu Lemma Institute of Pathobiology, Addis Ababa University for facilities support of the study. We would also like to thank Mojo health center laboratory staffs for provision of the necessary information, facilities and technical supports. We are indebted to data collectors for their cooperation during the field work.

\section{Funding}

Not applicable

\section{Availability of data and materials}

All data of findings are available from corresponding author on reasonable request. All relevant data are within the manuscript.

\section{Ethics approval and consent to participate}

Ethical clearance and support letter were obtained from Institutional review board (IRB) of Addis Ababa University, Aklilu Lemma Institute of Pathobiology, and Mojo health center to use the data for research purpose. No names or identifying information were indicated on the checklists, and confidentiality was assured throughout the study.

\section{Consent for publication}

Not applicable

\section{Competing interests}


The authors declare that they have no competing interests.

Author details

${ }^{1}$ Aklilu Lemma Institute of Pathobiology, Addis Ababa University, P.O.Box 1176, Addis Ababa, Ethiopia.

${ }^{2}$ College of Medicine and Health Sciences, Bahir Dar University, P.O.Box 79, Bahir Dar, Ethiopia

\section{References}

1. WHO: World malaria report 2020: 20 years of global progress and challenges. World Health Organization (WHO) 2020.

2. Beeson JG, Chu CS, Richards JS, Nosten F, Fowkes FJI: Plasmodium vivaxmalaria: Challenges in diagnosis, treatment and elimination. The Pediatric Infectious Disease Journal 2015, 34:529-531.

3. WWARN: Vivax malaria: The challenges towards malaria elimination. Worldwide antimalarial resistance network (WWARN) 2017.

4. Lover AA, Baird JK, Gosling R, Price RN: Malaria Elimination: Time to Target All Species.American Journal of Tropical Medicine and Hygiene 2018, 99:17-23.

5. WHO: World malaria report 2016. World Health Organization (WHO) 2016.

6. Price RN, Commons RJ, Battle KE, Thriemer K, Mendis K: Plasmodium vivaxin the Era of the Shrinking P. falciparum Map.Trends in Parasitology 2020, 36:560-570.

7. EPHI: Ethiopia national malaria indicator survey 2015. Addis Ababa, Ethiopia: Ethiopian Public Health Institute (EPHI); 2016.

8. Berhe B, Mardu F, Legese H, Negash H: Seasonal distribution and seven year trend of malaria in North West Tigrai: 2012-2018, Ethiopia; 2019. Tropical diseases, travel medicine and vaccines 2019, 5:1-7.

9. Shamebo T, Petros B: Trend analysis of malaria prevalence in Halaba special district, Southern Ethiopia.BMC research notes 2019, 12:190.

10. Addisu A, Tegegne Y, Mihiret Y, Setegn A, Zeleke AJ: A 7-year trend of malaria at primary health facilities in Northwest Ethiopia.Journal of parasitology research 2020, 2020.

11. USAID/CDC: President's malaria initiative Ethiopia-malaria operational plan FY 2017. United States of America Department of State (USAID)/Center for diseases control and prevention (CDC); 2017.

12. Deribew A, Dejene T, Kebede B, Tessema GA, Melaku YA, Misganaw A, Gebre T, Hailu A, Biadgilign S, Amberbir A: Incidence, prevalence and mortality rates of malaria in Ethiopia from 1990 to 2015: analysis of the global burden of diseases 2015. Malaria journal 2017, 16:1-7.

13. Gebretsadik D, Feleke DG, Fiseha M: Eight-year trend analysis of malaria prevalence in Kombolcha, South Wollo, north-central Ethiopia: a retrospective study.Parasites \& vectors 2018, 11:1-6.

14. Girum T, Shumbej T, Shewangizaw M: Burden of malaria in Ethiopia, 2000-2016: findings from the Global Health Estimates 2016. Tropical diseases, travel medicine and vaccines 2019, 5:1-7. 
15. Alkadir S, Gelana T, Gebresilassie A: A five year trend analysis of malaria prevalence in Guba district, Benishangul-Gumuz regional state, western Ethiopia: a retrospective study.Tropical Diseases, Travel Medicine and Vaccines 2020, 6:1-7.

16. USAID/CDC: President's malaria initiative Ethiopia: Malaria operational plan FY 2019. United States Agency for International Development (USAID) /Center for diseases control and prevention (CDC) 2019.

17. FMOH: National malaria elimination road map.Federal Ministry of Health (FMOH) 2017.

18. Deutsch-Feldman M, Brazeau NF, Parr JB, Thwai KL, Muwonga J, Kashamuka M, Kitoto AT, Aydemir $\mathrm{O}$, Bailey JA, Edwards JK: Spatial and epidemiological drivers of Plasmodium falciparum malaria among adults in the Democratic Republic of the Congo.BMJ global health 2020, 5:e002316.

19. Iqbal J, Al-Awadhi M, Ahmad S: Decreasing trend of imported malaria cases but increasing influx of mixed P. falciparum and P. vivax infections in malaria-free Kuwait.PloS one 2020, 15:e0243617.

20. Haile D, Ferede A, Kassie B, Abebaw A, Million Y: Five-Year Trend Analysis of Malaria Prevalence in Dembecha Health Center, West Gojjam Zone, Northwest Ethiopia: A Retrospective Study.Journal of Parasitology Research 2020, 2020.

21. Gemechu T, Samuel A, Yewhalaw D: Ten years trend analysis of malaria prevalence and its correlation with climatic variables in Sibu Sire District, east Wollega zone, Oromia regional state, Western Ethiopia: a retrospective study.Science, Technology and Arts Research Journal 2015, 4:99105.

22. Hawaria D, Getachew H, Zhong G, Demissew A, Habitamu K, Raya B, Lee M-C, Yewhalaw D, Yan G: Ten years malaria trend at Arjo-Didessa sugar development site and its vicinity, Southwest Ethiopia: a retrospective study.Malaria journal 2019, 18:1-11.

23. Esayas E, Tufa A, Massebo F, Ahemed A, Ibrahim I, Dillu D, Bogale EA, Yared S, Deribe K: Malaria epidemiology and stratification of incidence in the malaria elimination setting in Harari Region, Eastern Ethiopia.Infectious Diseases of Poverty 2020, 9:1-12.

24. Bogale T, Asefa B: Magnitude of Malaria Infection in Chalia District, West Shoa Zone, Oromia Reginal State, Ethiopia.Journal of Health, Medicine and Nursing 2017, 35.

25. Yimer F, Animut A, Erko B, Mamo H: Past five-year trend, current prevalence and household knowledge, attitude and practice of malaria in Abeshge, south-central Ethiopia.Malaria Journal 2015, 14:1-11.

26. Kalil FS, Bedaso MH, Wario SK: Trends of Malaria Morbidity and Mortality from 2010 to 2017 in Bale Zone, Ethiopia: Analysis of Surveillance Data.Infection and Drug Resistance 2020, 13:4379.

27. Derbie A, Alemu M: Five years malaria trend analysis in Woreta health center, Northwest Ethiopia.Ethiopian journal of health sciences 2017, 27:465-472.

28. Feleke DG, Gebretsadik D, Gebreweld A: Analysis of the trend of malaria prevalence in Ataye, North Shoa, Ethiopia between 2013 and 2017. Malaria journal 2018, 17:1-6.

29. Solomon A, Kahase $D$, Alemayehu M: Trend of malaria prevalence in Wolkite health center: an implication towards the elimination of malaria in Ethiopia by 2030.Malaria journal 2020, 19:1-8. 
30. Hailemariam M, Gebre S: Trend analysis of malaria prevalence in Arsi Negelle health center, Southern Ethiopia.Journal of Infectious Diseases and Immunity 2015, 7:1-6.

31. Cáceres Carrera L, Victoria C, Ramirez JL, Jackman C, Calzada JE, Torres R: Study of the epidemiological behavior of malaria in the Darien Region, Panama. 2015-2017.PloS one 2019, 14:e0224508.

32. Saikia S, Kumari S, Sharma RK: A Retrospective Study Of Malaria Trends Of Ghagua Health Centre, Kamrup Metropolitan District, Assam, India.INTERNATIONAL RESEARCH JOURNAL OF PHARMACY 2017, 10(5).

33. Narathattil A, Desai A, Ferreira AM, Bicholkar A: Ten years trend analysis of malaria cases at rural health and training centre Mandur, Goa.International Journal of Community Medicine and Public Health 2020, 7(10):3976-3979.

34. Tangpukdee N, Duangdee C, Wilairatana P, Krudsood S: Malaria diagnosis: a brief review. The Korean journal of parasitology 2009, 47:93.

35. Chirebvu E, Chimbari MJ, Ngwenya BN: Assessment of risk factors associated with malaria transmission in Tubu village, northern Botswana.Malaria research and treatment 2014, 2014.

36. Cook J, Owaga C, Marube E, Baidjoe A, Stresman G, Migiro R, Cox J, Drakeley C, Stevenson JC: Risk factors for Plasmodium falciparum infection in the Kenyan Highlands: a cohort study. Transactions of The Royal Society of Tropical Medicine and Hygiene 2019, 113:152-159.

37. Essendi WM, Vardo-Zalik AM, Lo E, Machani MG, Zhou G, Githeko AK, Yan G, Afrane YA: Epidemiological risk factors for clinical malaria infection in the highlands of Western Kenya.Malaria journal 2019, 18:1-7.

38. Habyarimana F, Ramroop S: Prevalence and Risk Factors Associated with Malaria among Children Aged Six Months to 14 Years Old in Rwanda: Evidence from 2017 Rwanda Malaria Indicator Survey.International Journal of Environmental Research and Public Health 2020, 17:7975.

39. Ugwu CLJ, Zewotir T: Spatial distribution and sociodemographic risk factors of malaria in Nigerian children less than 5 years old.Geospatial Health 2020, 15.

40. Baird JK, Maguire JD, Price RN: Diagnosis and treatment of Plasmodium vivax malaria.Advances in parasitology 2012, 80:203-270.

41. Baird JK, Valecha N, Duparc S, White NJ, Price RN: Diagnosis and treatment of Plasmodium vivax malaria. The American journal of tropical medicine and hygiene 2016, 95:35-51.

42. Cheng Q, Cunningham J, Gatton ML: Systematic Review of Sub-microscopic P. vivax Infections: Prevalence and Determining Factors.PLoS Neglected Tropical Diseases 2015, 9.

43. Goldlust SM, Thuan PD, Giang DDH, Thang ND, Thwaites GE, Farrar J, Thanh NV, Nguyen TD, Grenfell BT, Boni MF, Hien TT: The decline of malaria in Vietnam, 1991-2014.Malaria Journal 2018, 17.

44. Jemal A, Ketema T: A declining pattern of malaria prevalence in Asendabo Health Center Jimma zone, Southwest Ethiopia. BMC Research Notes 2019, 12. 
45. Aregawi M, Lynch M, Bekele W, Kebede H, Jima D, Taffese HS, Yenehun MA, Lilay A, Williams R, Thomson M, et al: Time Series Analysis of Trends in Malaria Cases and Deaths at Hospitals and the Effect of Antimalarial Interventions, 2001-2011, Ethiopia.PLOS ONE 2014, 9:e106359.

46. Minwuyelet A, Aschale Y: Analysis of Five-Year Trend of Malaria at Bichena Primary Hospital, Amhara Region, Ethiopia Journal of Parasitology Research 2021.

47. Ferreira MU, Oliveira TCd: Challenges for Plasmodium vivax malaria elimination in the genomics era. Pathogens and Global Health 2015, 109:89-90

48. WHOa: Confronting Plasmodium vivax Malaria.World Health Organization (WHO) 2015.

49. WHOb: Control and elimination of Plasmodium vivax malaria. A technical brief.World Health Organization(WHO) 2015.

50. Baird JK: Chloroquine Resistance inPlasmodium vivax.ANTIMICROBIALAGENTS ANDCHEMOTHERAPY 2004, 48:4075-4083

51. Anstey NM, Russell B, Yeo TW, Price RN: The pathophysiology of vivax malaria.Trends in Parasitology 2009, 25:220-227.

52. Hossain MS, Commons RJ, Douglas NM, Thriemer K, Alemayehu BH, Amaratunga C, Anvikar AR, Ashley EA, Asih PBS, Carrara V, et al: The risk of Plasmodium vivax parasitaemia after P.falciparum malaria: An individual patient data meta-analysis from the World Wide Antimalarial Resistance Network.PLoS Medicine 2020, 17:e1003393.

53. Kendjo E, Houzé S, Mouri O, Taieb A, Gay F, Jauréguiberry S, Tantaoui I, Ndour PA, Buffet P, Piarroux $\mathrm{M}$, et al: Epidemiologic Trends in Malaria Incidence Among Travelers Returning to Metropolitan France, 1996-2016.Journal of American Medical Association Network Open 2019, 2.

54. UNDP: DISCUSSION PAPER: GENDER AND MALARIA.United Nations Development Programme (UNDP) 2015.

55. Steeg LGv, Flores-Garcia Y, Zavala F, Klein SL: Irradiated sporozoite vaccination induces sex-specific immune responses and protection against malaria in mice. Vaccine 2019, 37:4468-4476.

56. Briggs J, Teyssier N, Nankabirwa Jl, Rek J, Jagannathan P, Arinaitwe E, Bousema T, Drakeley C, Murray M, Crawford E, et al: Sex-based differences in clearance of chronic Plasmodium falciparum infection.elife 2020, 9.

\section{Figures}




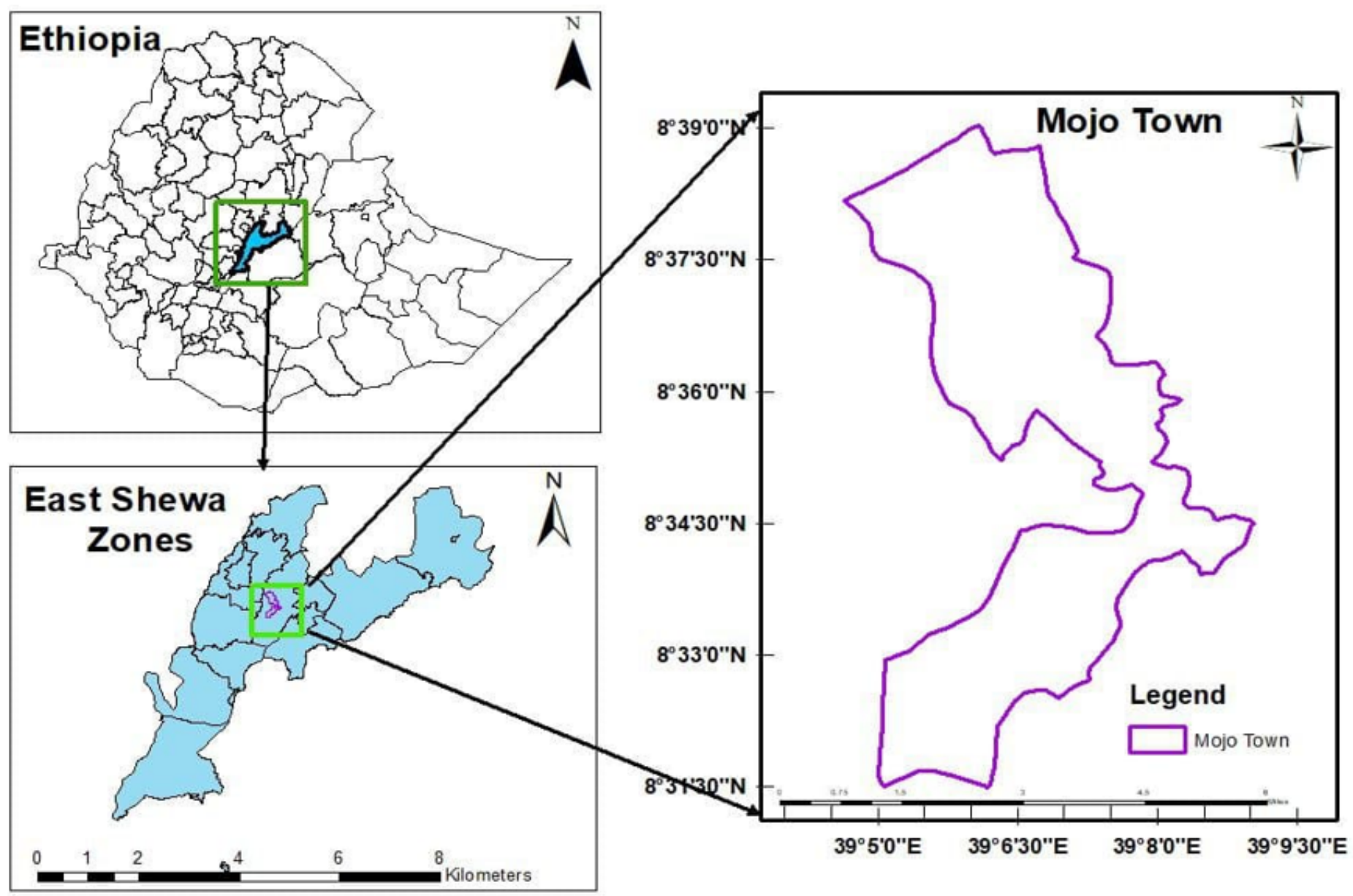

Figure 1

Physical location of Mojo town 


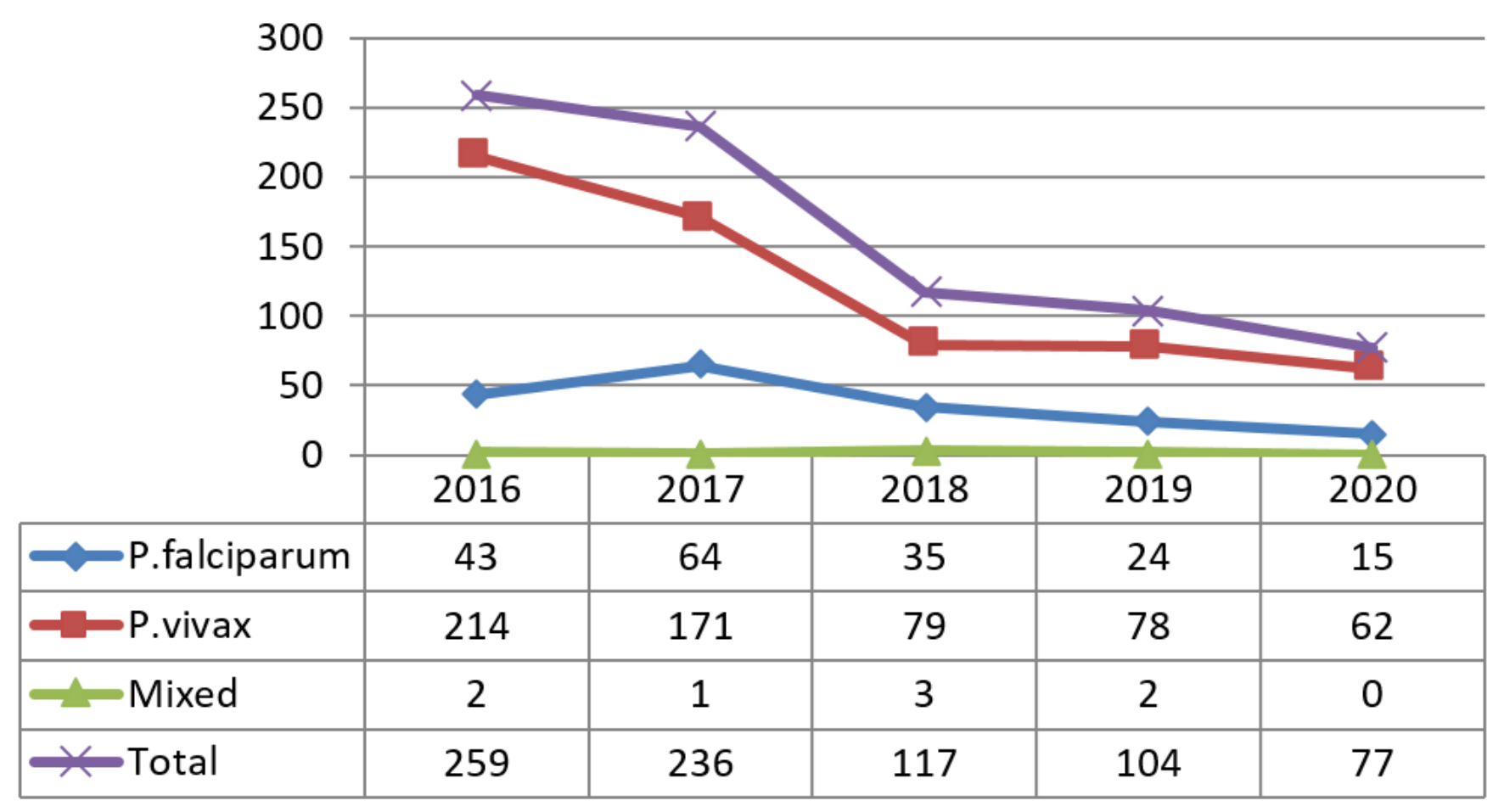

Figure 2

Annual trends of malaria cases in Mojo health center from 2016 to 2020

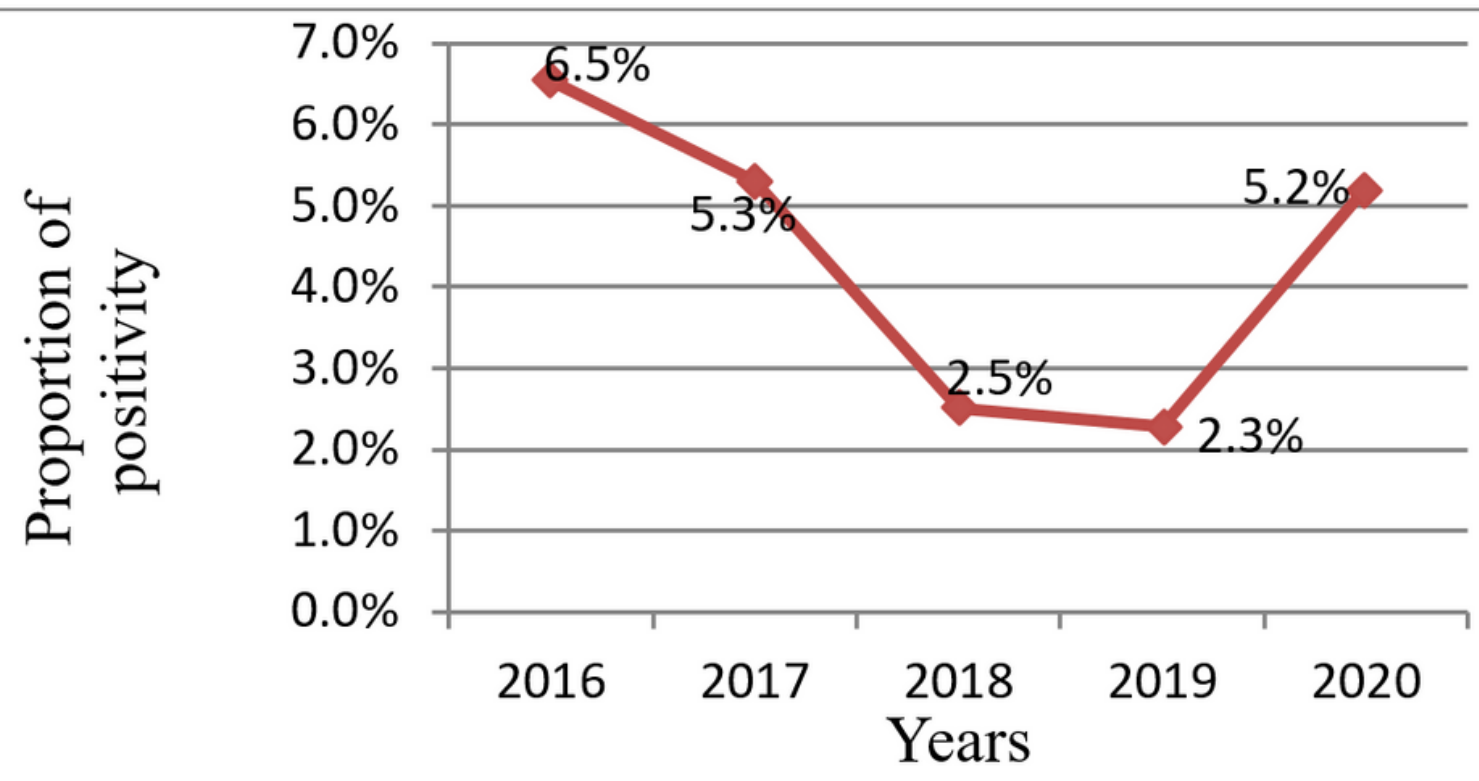

Figure 3

Annual trends of malaria prevalence in Mojo health center from 2016 to 2020 


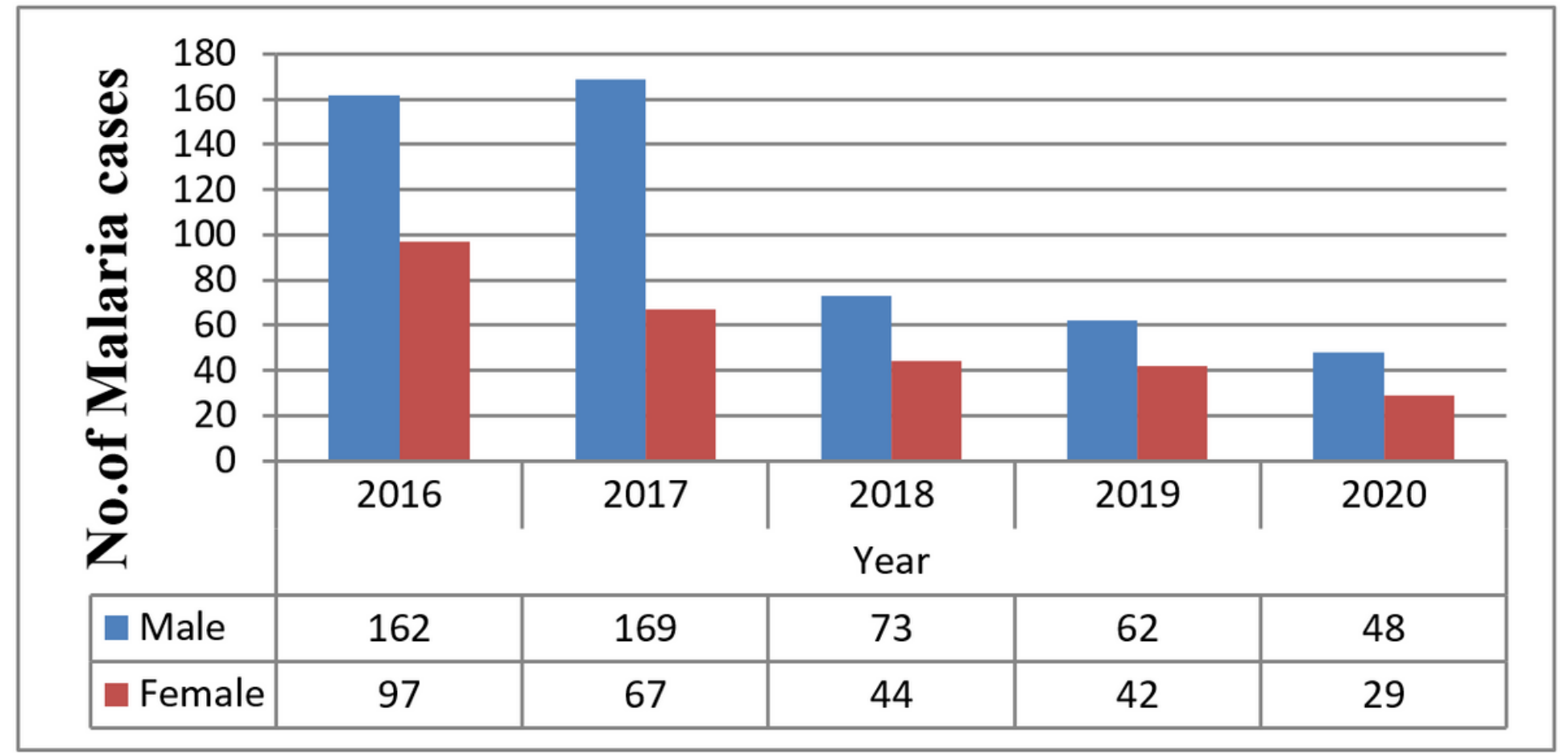

Figure 4

Annual trend of malaria cases by sex in in Mojo health center from 2016 to 2020

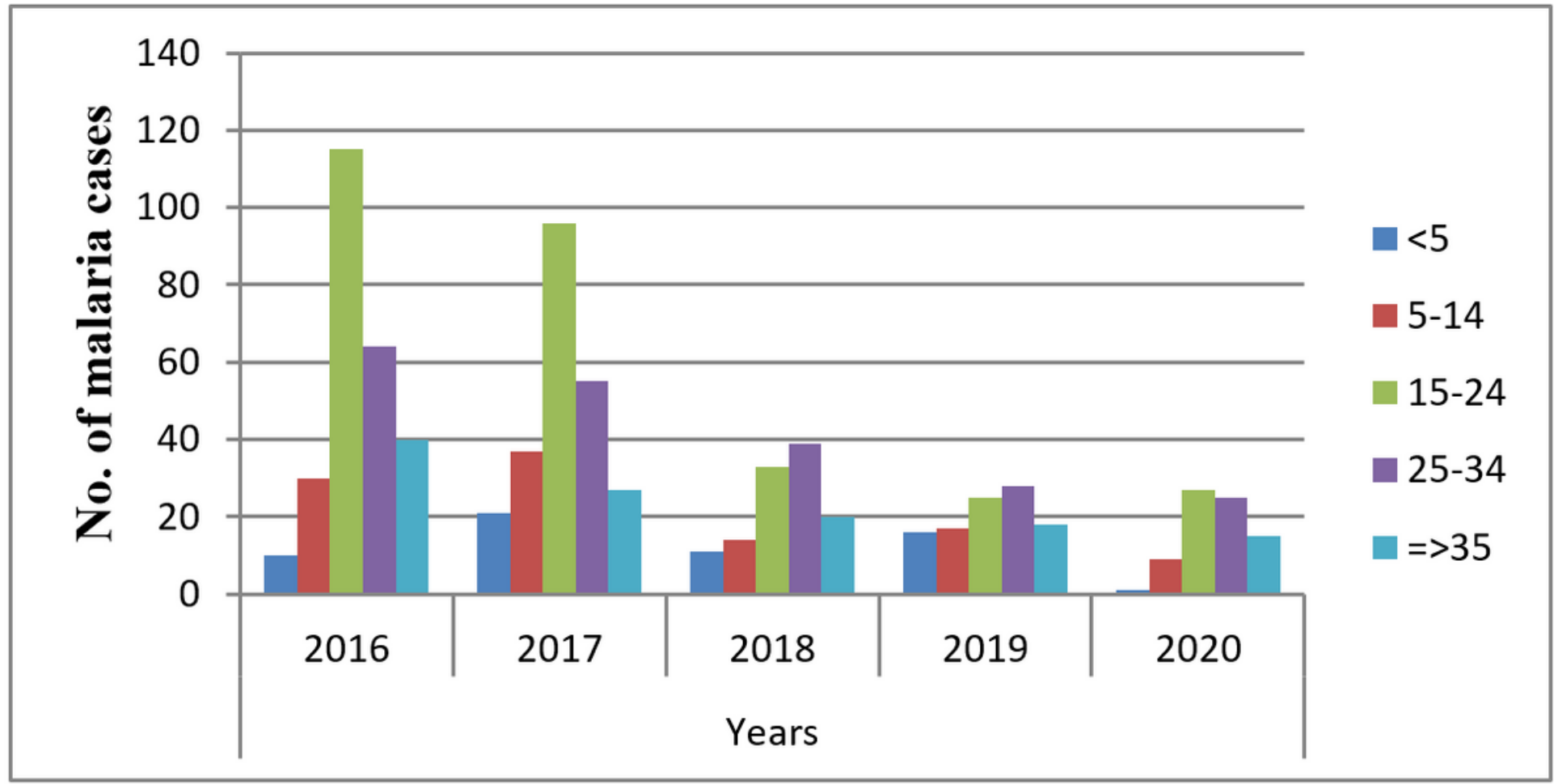

Figure 5

Annual trend of malaria cases by age in Mojo health center from 2016 to 2020 


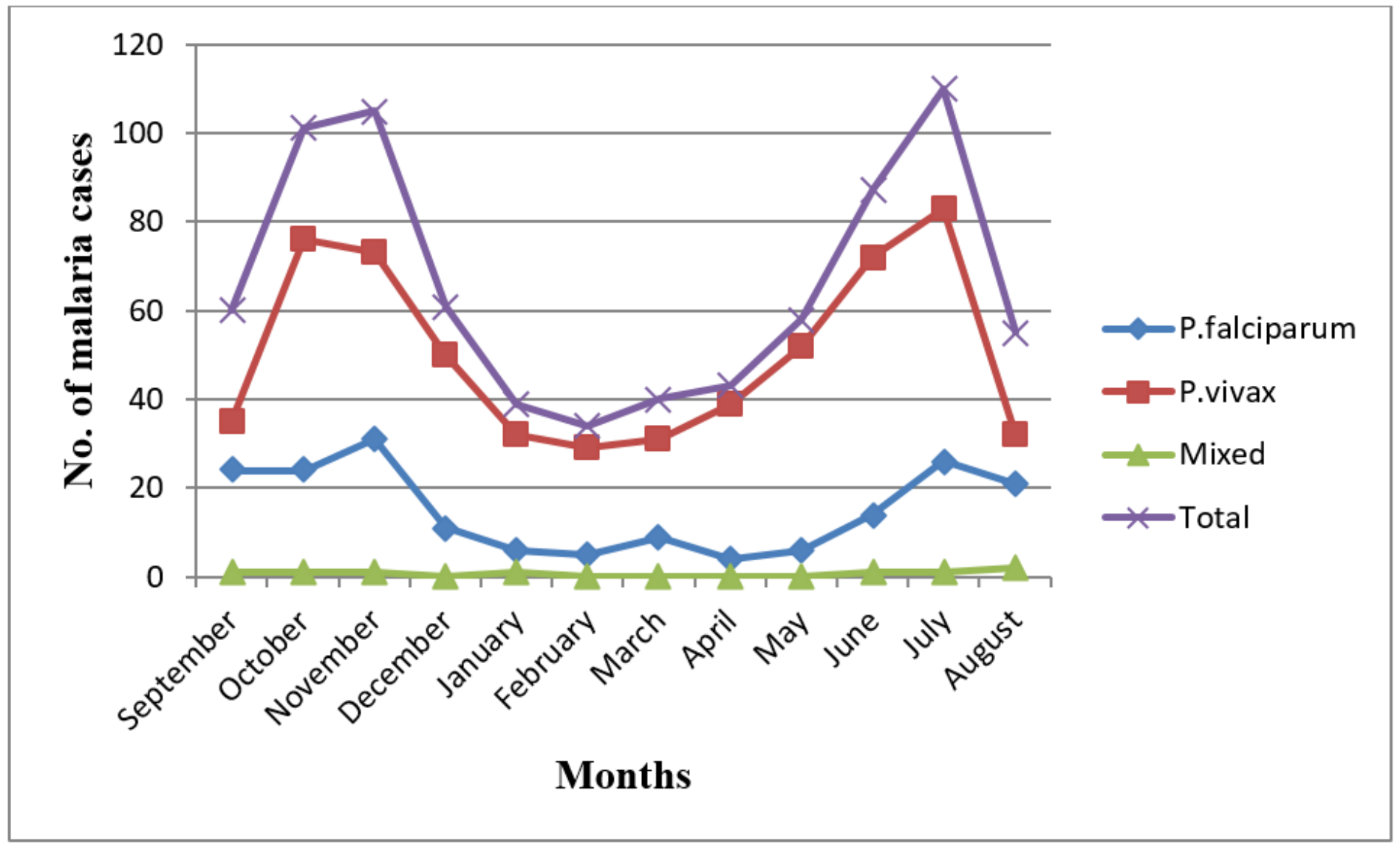

Figure 6

Trend of malaria prevalence with Plasmodium species and month in Mojo health center from 2016 to 2020

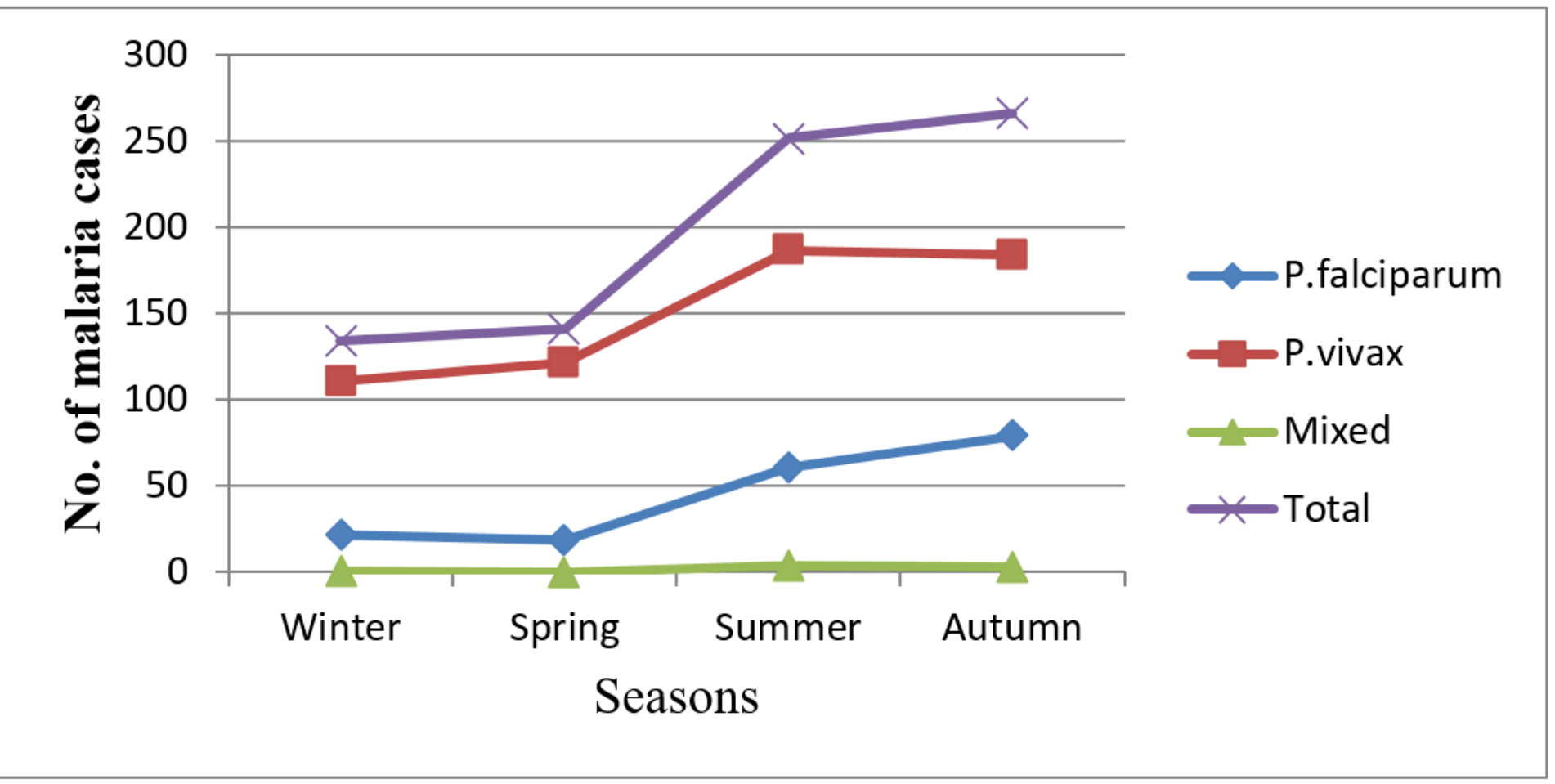


Figure 7

Seasonal profile of P. vivax, P. falciparum and mixed infections in Mojo health center from 2016 to 2020

\section{Supplementary Files}

This is a list of supplementary files associated with this preprint. Click to download.

- Graphicalabstract.docx 